\title{
Convalescent Plasma Therapy and Its Effects On COVID-19 Patient Outcomes: A Systematic Review of Current Literature
}

\author{
Nabiyah Bakhtawar ${ }^{1}$, Muhammad Usman ${ }^{2,3}$, Malik Muhammad Uzair Khan ${ }^{4}$ \\ 1. Internal Medicine, University Hospital Coventry and Warwickshire, Coventry, GBR 2. Internal Medicine, Kettering \\ General Hospital, Kettering, GBR 3. Internal Medicine, Leicester Royal Infirmary, Leicester, GBR 4. Department of \\ Anatomy, Shalamar Medical and Dental College, Lahore, PAK
}

Corresponding author: Nabiyah Bakhtawar, biyabakhtawar@gmail.com

\begin{abstract}
Started in late 2019, coronavirus disease 2019 (COVID-19) has rapidly turned into a global pandemic. Considering there is no proven therapy for COVID-19 infection, there is a need to propose potential treatment options. The use of convalescent plasma is one such option as convalescent plasma has previously been used for treating outbreaks of Ebola, influenza, Middle East Respiratory Syndrome Coronavirus (MERS$\mathrm{CoV}$ ), and severe acute respiratory (SAR) viruses. Therefore, we carried out an early systematic review to evaluate the efficacy of convalescent plasma (CP) therapy and its effects on COVID-19 patient outcomes. A structured and rigorous systematic review was carried out that included all studies conducted on this topic between December 2019 and June 2020. A total of 10 studies containing a mix of case reports, case series, observational studies, and randomized control trials were identified. Most of the studies lacked randomization and included only small groups of patients. Considering the limitations in the design of current studies, it is difficult to draw a definitive conclusion. However, our results showed that plasma therapy produces notable improvements in patients' clinical symptoms and radiological and biochemical parameters associated with COVID-19 infection. Based on the available information, it is difficult to draw a tangible conclusion about whether plasma therapy improves patient mortality. Until we have concrete evidence to prove otherwise, convalescent plasma therapy may be used as adjuvant therapy for treating COVID-19 infection in critically ill patients.
\end{abstract}

Categories: Infectious Disease, Pulmonology, Hematology

Keywords: convalescent plasma therapy, convalescent plasma, corona virus, covid 19

\section{Introduction And Background}

The coronavirus disease 2019 (COVID-19) has turned into a rapidly evolving pandemic. As of 13th July 2020, the World Health Organization (WHO) has confirmed that the number of COVID-19 cases has reached $12,768,307$, and the recorded death toll has crossed 566,654 [1]. WHO estimates that the COVID-19 related mortality curve will level off at $5.7 \%$ [2].

Received 07/14/2020 Review began $07 / 15 / 2020$ Review ended 07/17/2020 Published 08/03/2020

(c) Copyright 2020

Bakhtawar et al. This is an open access article distributed under the terms of the Creative Commons Attribution License CC-BY 4.0, which permits unrestricted use, distribution, and reproduction in any medium, provided the original author and source are credited.
Despite the desperate attempts, the treatment for COVID-19 is largely symptomatic. Currently, there are no proven treatments for COVID-19 [3].

Convalescent blood products include whole blood, plasma, serum, and isolates such as immunoglobulins and antibodies. These products are gathered from a patient who has already recovered from an infection and is a possible human source of specific antibodies [4].

Convalescent plasma has previously shown clinical efficacy in other virus-borne infections. WHO recommended the use of convalescent plasma from recovered patients for empirical treatment during the Ebola outbreak [5]. During the 2019 influenza A virus subtype H1N1 pandemic, the use of convalescent plasma therapy by Hung et al. showed a significant reduction in mortality rates in the treatment group compared to control (20.0\% vs. $54.8 \%$; $=0.01$ ) [6]. Convalescent plasma therapy has also shown benefit in the treatment of Middle East Respiratory Syndrome Coronavirus (MERS-CoV), and severe acute respiratory infections (SAR) viruses [7, 8]. Several randomized control trials are underway to determine the efficacy of convalescent plasma therapy for COVID-19 infection [9].

There is a lack of structured systematic reviews looking into the efficacy of convalescent plasma therapy for COVID-19 patients. Therefore, we have conducted this early systematic review to provide an insight into the clinical effectiveness of convalescent plasma as a potential therapy for COVID-19 patients.

\section{Review}

Methods 
Two independent reviewers (Bakhtawar Nabiyah [BN] and Usman Muhammad [UM]) carried out a literature review using the Preferred Reporting Items for Systematic Reviews and Meta-Analyses (PRISMA) guidelines for a systematic review. This was followed by an independent evaluation of the extracted data by Khan Malik Uzair (KM). We used electronic databases such as PubMed ${ }^{\circledR}$, Embase ${ }^{\circledR}$, Google Scholar, Cochrane Library, and MEDLINE ${ }^{\circledR}$ to look for case reports, case series, observational studies, and randomized control trials conducted between December 2019 and June 2020. Two search themes were used for literature review and were joined using the Boolean operator "AND". For the theme "COVID", we used keywords such as "coronavirus", "COVID-19", and "SARS-COV-2". For the theme "convalescent plasma", we used "convalescent plasma" and "plasma therapy" as the main keywords.

Inclusion Criteria

We included all articles published between December 2019 and June 2020. We included case series, case reports, observational studies, and randomized control trials. We only included full-text manuscripts available in the English language.

Exclusion Criteria

We excluded review articles, commentaries, notes to editors, and all other articles in which convalescent plasma therapy was not used as a treatment option. We also excluded studies published in languages other than English for which there were no available translated manuscripts.

Data Extraction and Study Selection

$\mathrm{BN}$ and UM carried out a rigorous literature review independently. KM then independently evaluated the results from both the researchers. Once the literature review was complete, the researchers compiled and compared their results for any conflicts that were resolved through mutual consultation.

A total of 156 studies were identified following the initial literature review. The reviewers used 17 studies after excluding duplicate studies and after reading through the titles, abstracts, and methodologies of the studies. They used 10 studies for their final analysis.

Figure 1 describes the literature review process in detail.
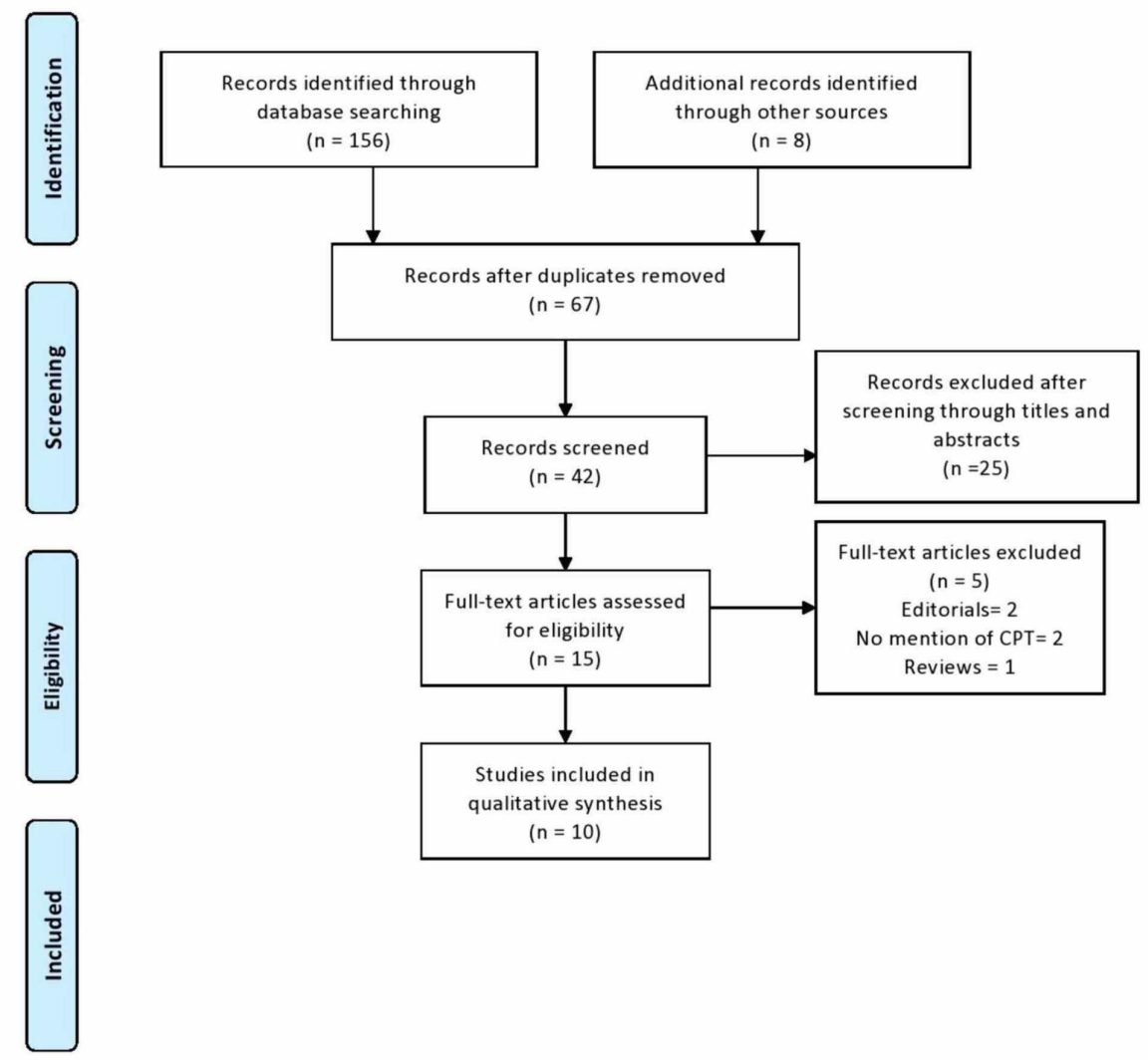


\section{Cureus}

\section{FIGURE 1: Flowchart describing study identification and selection}

process

CPT - convalescent plasma therapy

\section{Results}

A total of 10 studies were included in this systematic review [10-19]. We were able to identify five case series $[10,12,13,15,16]$, two case reports [14, 17], one prospective observational study [11], one retrospective observational study [18], and one randomized control trial [19]. These studies included a total of 156 patients with a mean age between 28 and 73 years. Table 1 describes the patient demographics in detail.

\begin{tabular}{|c|c|c|c|c|c|c|c|c|}
\hline Author & Study type & $\begin{array}{l}\text { Number of } \\
\text { patients }\end{array}$ & $\begin{array}{l}\text { Median } \\
\text { age (years) }\end{array}$ & Gender & $\begin{array}{l}\text { Disease } \\
\text { severity }\end{array}$ & $\begin{array}{l}\text { Time of } \\
\text { administration } \\
\text { of plasma } \\
\text { therapy }\end{array}$ & $\begin{array}{l}\text { Intervention } \\
\text { used }\end{array}$ & Concurrent treatment \\
\hline $\begin{array}{l}\text { Ahn et } \\
\text { al. } \\
2020 \\
\text { [10] }\end{array}$ & Case series & 2 & $\begin{array}{l}\text { Male: } 71 \\
\text { Female: } \\
67\end{array}$ & $\begin{array}{l}\text { Male: } 1 \\
\text { Female: } 1\end{array}$ & Severe & $\begin{array}{l}\text { Plasma used } \\
\text { on day } 7 \text { (case } \\
2 \text {, female) and } \\
\text { day } 22 \text { (case } \\
1 \text {, male) of } \\
\text { presentation }\end{array}$ & $\begin{array}{l}500 \mathrm{ml} \\
\text { plasma used } \\
\text { in two } \\
\text { divided } \\
\text { doses }\end{array}$ & $\begin{array}{l}\text { Antiviral therapy: lopinavir/ritonavir } 400 \mathrm{mg} / 100 \mathrm{mg} \text { PO BD. } \\
\text { Steroids: methylprednisolone } 0.5 / 1 \mathrm{mg} / \mathrm{kg} / \text { day IV daily. } \\
\text { Empirical antibiotics hydroxychloroquine: } 400 \mathrm{mg} \text { PO OD. } \\
\text { Oxygen therapy: intubation and mechanical ventilator care. }\end{array}$ \\
\hline $\begin{array}{l}\text { Duan } \\
\text { et al. } \\
2020 \\
{[11]}\end{array}$ & $\begin{array}{l}\text { Prospective } \\
\text { observational } \\
\text { study }\end{array}$ & 10 & 52.5 & $\begin{array}{l}\text { Males: } 6 \\
\text { Females: } 4\end{array}$ & Severe & $\begin{array}{l}\text { Between } 10 \\
\text { and } 20 \text { days of } \\
\text { presentation, } \\
\text { median } \\
\text { administration } \\
\text { time } 16.7 \text { days }\end{array}$ & $\begin{array}{l}200 \mathrm{ml} \\
\text { convalescent } \\
\text { plasma with } \\
\text { an antibody } \\
\text { titer }>1: 640 \\
\text { given as one } \\
\text { dose }\end{array}$ & $\begin{array}{l}\text { Antiviral therapy: ribavirin } 0.5 \mathrm{~g} \text { per day IV, or peramivir } 0.3 \\
\mathrm{~g} \text { per day IV, or oseltamivir } 75 \mathrm{mg} \text { PO BD, or arbidol } 0.2 \mathrm{~g} \\
\text { PO TDS as a monotherapy or in combination therapy with } \\
\text { peramivir } 0.3 \mathrm{~g} \text { per day IV, or remdesivir } 0.2 \mathrm{~g} \text { per day IV, } \\
\text { or oseltamivir } 75 \mathrm{mg} \mathrm{PO} \mathrm{BD} \text {, or ribavirin } 0.5 \mathrm{~g} \text { per day IV. } \\
\text { Steroids: methylprednisolone } 20 \mathrm{mg} \text { IV daily. Empirical } \\
\text { antibiotics and anti-fungals. Oxygen therapy: mechanical } \\
\text { ventilation, or oxygenation via high-flow nasal cannula or } \\
\text { low-flow nasal cannula. }\end{array}$ \\
\hline $\begin{array}{l}\text { Pei et } \\
\text { al. } \\
2020 \\
{[12]}\end{array}$ & Case series & $\begin{array}{l}3 \text { (one } \\
\text { patient } \\
\text { developed } \\
\text { anaphylaxis } \\
\text { and } \\
\text { dropped } \\
\text { out) }\end{array}$ & $\begin{array}{l}\text { Not } \\
\text { mentioned }\end{array}$ & $\begin{array}{l}\text { Not } \\
\text { mentioned }\end{array}$ & $\begin{array}{l}\text { Moderate } \\
\text { to severe }\end{array}$ & $\begin{array}{l}\text { Between } 12 \\
\text { and } 27 \text { days of } \\
\text { hospital } \\
\text { admission }\end{array}$ & $\begin{array}{l}200-400 \mathrm{ml} \\
\text { antibody titer } \\
1: 160 \text { given } \\
\text { as one dose }\end{array}$ & Not mentioned in detail. \\
\hline $\begin{array}{l}\text { Shen } \\
\text { et al. } \\
2020 \\
{[13]}\end{array}$ & Case series & 5 & $36-65$ & $\begin{array}{l}\text { Males: } 3 \\
\text { Females: } 2\end{array}$ & Severe & $\begin{array}{l}\text { Between } 10 \\
\text { and } 22 \text { days of } \\
\text { admission }\end{array}$ & $\begin{array}{l}200-250 \mathrm{ml} \\
\text { plasma with } \\
\text { an antibody } \\
\text { titer }>1: 1000 \\
\text { given as two } \\
\text { doses }\end{array}$ & $\begin{array}{l}\text { Antiviral therapy: darunavir, ritonavir/lopinavir, arbidol, } \\
\text { interferon alfa-1b, or favipiravir. Steroids: } \\
\text { methylprednisolone. Empirical antibiotics and anti-fungals. } \\
\text { Oxygen therapy: mechanical ventilation. }\end{array}$ \\
\hline $\begin{array}{l}\text { Tan et } \\
\text { al. } \\
2020 \\
{[14]}\end{array}$ & Case report & 1 & $\begin{array}{l}\text { Not } \\
\text { mentioned }\end{array}$ & Male: 1 & Moderate & $\begin{array}{l}\text { On 48th day of } \\
\text { admission }\end{array}$ & $\begin{array}{l}400 \mathrm{ml} \\
\text { plasma } \\
\text { (doses and } \\
\text { antibody titer } \\
\text { not } \\
\text { mentioned) }\end{array}$ & Not mentioned in detail. \\
\hline $\begin{array}{l}\text { Ye et } \\
\text { al. } \\
2020 \\
{[15]}\end{array}$ & Case series & 6 & $28-75$ & $\begin{array}{l}\text { Males: } 3 \\
\text { Females: } 3\end{array}$ & Severe & $\begin{array}{l}\text { One dose } \\
\text { given }>30 \text { days } \\
\text { after } \\
\text { admission on } \\
\text { average }\end{array}$ & $\begin{array}{l}200 \mathrm{ml} \\
\text { plasma given } \\
\text { in 1-3 doses } \\
\text { (antibody } \\
\text { titer not } \\
\text { reported) }\end{array}$ & $\begin{array}{l}\text { Antiviral therapy: arbidol. Empirical antibiotics: ofloxacin in } \\
\text { one patient. Oxygen therapy. }\end{array}$ \\
\hline & & & & & & & $\begin{array}{l}\text { Plasma was } \\
\text { given in a }\end{array}$ & \\
\hline
\end{tabular}




\section{Cureus}

\begin{tabular}{|c|c|c|c|c|c|c|c|c|}
\hline $\begin{array}{l}\text { Zhang } \\
\text { et al. } \\
2020 \\
{[16]}\end{array}$ & Case series & 4 & $31-73$ & $\begin{array}{l}\text { Males: } 2 \\
\text { Females: } 2\end{array}$ & Severe & $\begin{array}{l}\text { Between } 11 \\
\text { and } 41 \text { days of } \\
\text { admission }\end{array}$ & $\begin{array}{l}\text { dose range } \\
\text { of } 200-2400 \\
\mathrm{~mL} \text {; given in } \\
1-8 \text { doses } \\
\text { (antibody } \\
\text { titer not } \\
\text { reported) }\end{array}$ & $\begin{array}{l}\text { Antiviral therapy: different anti-virals including lopinavir- } \\
\text { ritonavir, interferon-alpha, arbidol, oseltamivir, and } \\
\text { ribavirin. Steroids: methylprednisolone. Empirical } \\
\text { antibiotics and anti-fungals. Oxygen therapy: mechanical } \\
\text { ventilation, high-flow nasal oxygen, ECMO. }\end{array}$ \\
\hline $\begin{array}{l}\text { Zhang } \\
\text { et al. } \\
2020 \\
{[17]}\end{array}$ & Case report & 1 & 64 & Female: 1 & Severe & $\begin{array}{l}\text { On day } 17 \text { of } \\
\text { hospitalization }\end{array}$ & $\begin{array}{l}200 \mathrm{ml} \text { with } \\
\text { antibody titer } \\
1: 160 \text { (no. of } \\
\text { doses not } \\
\text { mentioned) }\end{array}$ & Not mentioned in detail. \\
\hline $\begin{array}{l}\text { Zeng } \\
\text { et al. } \\
2020 \\
{[18]}\end{array}$ & $\begin{array}{l}\text { Retrospective } \\
\text { observational } \\
\text { study }\end{array}$ & $\begin{array}{l}21 \\
\text { (treatment } \\
\text { group 6, } \\
\text { control } \\
\text { group 15) }\end{array}$ & $\begin{array}{l}\text { Treatment } \\
\text { group: } \\
61.3 . \\
\text { Control } \\
\text { group: } 73\end{array}$ & $\begin{array}{l}\text { Treatment } \\
\text { group: } \\
\text { males 5, } \\
\text { female 1. } \\
\text { control } \\
\text { group: } \\
\text { males 11, } \\
\text { females } 4\end{array}$ & Severe & $\begin{array}{l}\text { Median } 21.5 \\
\text { days of } \\
\text { hospitalization }\end{array}$ & $\begin{array}{l}300 \mathrm{ml} \\
\text { plasma given } \\
\text { as two } \\
\text { doses to } \\
\text { three } \\
\text { patients and } \\
\text { one dose to } \\
\text { three } \\
\text { patients }\end{array}$ & Not mentioned in detail. \\
\hline $\begin{array}{l}\text { Li et al. } \\
2020 \\
{[19]}\end{array}$ & $\begin{array}{l}\text { Randomized } \\
\text { control trial }\end{array}$ & $\begin{array}{l}103 \\
\text { (treatment } \\
\text { group 52, } \\
\text { control 51) }\end{array}$ & $\begin{array}{l}\text { Treatment } \\
\text { group: } 70 . \\
\text { Control } \\
\text { group: } 69\end{array}$ & $\begin{array}{l}\text { Treatment } \\
\text { group: } \\
\text { males } 27, \\
\text { female } 25 . \\
\text { Control } \\
\text { group: } \\
\text { males } 33, \\
\text { females } 18\end{array}$ & $\begin{array}{l}\text { Severe or } \\
\text { life- } \\
\text { threatening } \\
\text { COVID-19 }\end{array}$ & $\begin{array}{l}\text { Median } 27 \text { day } \\
\text { of } \\
\text { hospitalization }\end{array}$ & $\begin{array}{l}\text { Plasma was } \\
\text { given in a } \\
\text { dose range } \\
\text { of } 4 \text { to } 13 \\
\mathrm{~mL} / \mathrm{kg} \text { and } \\
\text { antibody titer } \\
1: 640 \\
\text { (number of } \\
\text { doses not } \\
\text { clear) }\end{array}$ & Not mentioned in detail. \\
\hline
\end{tabular}

TABLE 1: Summary of study type, patient demographics, plasma therapy intervention, and concurrent treatment modalities

PO - by mouth; OD - once daily; BD - twice daily; ECMO - extracorporeal membrane oxygenation

All the studies included patients ranging from moderate COVID-19 infection to severe and life-threatening infections. The patients in the studies received plasma therapy between day seven to day 48 of their hospital admission [10-19]. All the studies used varying doses, frequency of administration, and plasma with varying antibody titers. Duan et al. used $200 \mathrm{ml}$ convalescent plasma in one dose [11]. Whereas, Zhang et al. used up to $2,400 \mathrm{ml}$ plasma in up to eight divided doses [16]. Furthermore, most of the studies reported a variety of concurrent treatments such as antivirals, antibiotics, steroids, antimalarial, anti-fungal, and a variety of modalities for oxygen therapy (ranging from the nasal cannula to mechanical ventilation and extracorporeal membrane oxygenation [ECMO]) [10-19] (Table 1).

Most of the studies reported patient mortalities on follow-up, and almost all patients were alive at the time of follow-up in some studies [10-13, 15-16]. In the study by Zeng at al., five out of six patients died despite receiving plasma therapy [18]. Similarly, Li et al. did not report any difference in mortalities in the treatment vs. control group on the 28th day of follow-up (15.7\% vs. $24.0 \%$; $p=0.30$ ) [19]. Most of the studies reported a reduction in viral shedding with the viral load turning negative following plasma therapy [10-16, 18-19].

The duration of discharge varied from as little as four days following CP therapy to as much as 35 days following CP therapy $[13,15]$. However, Li et al. did not report any difference in the time of discharge following $\mathrm{CP}$ in treatment vs. control groups (51.0\% in treatment vs $36.0 \%$ in the control group on day 28 of follow-up; $\mathrm{p}=0.120)$ [19].

As for the laboratory parameters, studies showed improvement in C-reactive protein (CRP) [10, 11, 13], interleukin 6 (IL-6) [10, 13], white cell count and/or lymphopaenia [10, 11], procalcitonin [13], and SARSCOV-2 immunoglobulin G (IgG) and immunoglobulin M (IgM) titers [15].

Ahn et al. reported a reduction in fever [10], and six studies reported an improvement in the demand for 


\section{Cureus}

oxygen [10, 11, 13, 15-17]. However, the randomized controlled trials (RCT) by Li et al. did not report any statistically significant difference in clinical improvement in the CP vs. control group on the 28th day of follow-up (51.9\% on convalescent plasma group showed clinical improvement vs. 43.1 in the control group; $\mathrm{p}=0.26)[19]$.

Ahn et al. reported improvement in pulmonary infiltrates as noted on chest X-ray [10]. Three more studies reported improvement in pulmonary infiltrates on repeat CT scans of the chest $[11,15,16]$.

Table 2 describes the effects of $\mathrm{CP}$ therapy on patient outcomes in detail.

\begin{tabular}{|c|c|c|c|c|c|c|c|}
\hline & $\begin{array}{l}\text { All-cause } \\
\text { mortality }\end{array}$ & $\begin{array}{l}\text { Duration of } \\
\text { discharge from } \\
\text { hospital after } \\
\text { plasma therapy }\end{array}$ & $\begin{array}{l}\text { Patients } \\
\text { discharged } \\
\text { from ITU } \\
\text { following } \\
\text { plasma } \\
\text { therapy at the } \\
\text { time of follow- } \\
\text { up }\end{array}$ & $\begin{array}{l}\text { Improvement in } \\
\text { laboratory parameters }\end{array}$ & $\begin{array}{l}\text { Improvement in clinical } \\
\text { parameters }\end{array}$ & $\begin{array}{l}\text { Improvement } \\
\text { in } \\
\text { radiological } \\
\text { parameters }\end{array}$ & $\begin{array}{l}\text { Improvement in } \\
\text { viral load }\end{array}$ \\
\hline $\begin{array}{l}\text { Ahn et } \\
\text { al. } \\
2020 \\
\text { [10] }\end{array}$ & $\begin{array}{l}\text { Both patients } \\
\text { alive at the } \\
\text { time of } \\
\text { follow-up }\end{array}$ & 18 days & Not reported & $\begin{array}{l}\text { Case 1: improvement } \\
\text { in CRP and IL-6 to } \\
\text { normal. Case 2: } \\
\text { improvement in CRP, } \\
\text { IL-6, and lymphopenia. }\end{array}$ & $\begin{array}{l}\text { Case 1: fever and oxygen } \\
\text { demands subsided. Case 2: } \\
\text { significant improvement in } \\
\text { oxygen demands. }\end{array}$ & $\begin{array}{l}\text { Case 1: } \\
\text { improvement } \\
\text { in X-ray } \\
\text { pulmonary } \\
\text { infiltrates. } \\
\text { Case 2: } \\
\text { improvement } \\
\text { in X-ray } \\
\text { pulmonary } \\
\text { infiltrates. }\end{array}$ & $\begin{array}{l}\text { Case 1: reduction in } \\
\text { SARS-CoV-2 RNA } \\
\text { by rRT-PCR. Case } \\
\text { 2: complete } \\
\text { recovery with no } \\
\text { detectable SARS- } \\
\text { CoV-2 RNA by rRT- } \\
\text { PCR. }\end{array}$ \\
\hline $\begin{array}{l}\text { Duan } \\
\text { et al. } \\
2020 \\
{[11]}\end{array}$ & $\begin{array}{l}\text { All patients } \\
\text { alive at the } \\
\text { time of } \\
\text { follow-up }\end{array}$ & Not reported & Not reported & $\begin{array}{l}\text { mean } 55.98 \text { before } \mathrm{CP} \\
\text { therapy to } 18.13 \text { after } \\
\mathrm{CP} \text { therapy; } \\
\text { improvement in } \\
\text { lymphocytopenia from } \\
\text { a mean } 0.65 \text { before } \mathrm{CP} \\
\text { transfusion to } 0.76 \\
\text { after therapy. }\end{array}$ & $\begin{array}{l}\text { Improvement in oxygen } \\
\text { saturation from mean } 93 \% \\
\text { before CP therapy to } 96 \% \\
\text { after therapy. }\end{array}$ & $\begin{array}{l}\text { CT chest for } \\
\text { showed } \\
\text { improvement } \\
\text { in pulmonary } \\
\text { infiltrates } \\
\text { following CP } \\
\text { therapy. }\end{array}$ & $\begin{array}{l}\text { All patients } \\
\text { detected negative } \\
\text { for SARS-CoV-2 } \\
\text { RNA by rRT-PCR } \\
\text { following CP } \\
\text { therapy. }\end{array}$ \\
\hline $\begin{array}{l}\text { Pei et } \\
\text { al. } \\
2020 \\
{[12]}\end{array}$ & $\begin{array}{l}\text { All patients } \\
\text { alive at the } \\
\text { time of } \\
\text { follow-up }\end{array}$ & $\begin{array}{l}6,14,23 \text { days for } \\
\text { three patients }\end{array}$ & All discharged & Not reported & Not clearly mentioned & Not reported & $\begin{array}{l}\text { Two patients had } \\
\text { negative viral load } \\
\text { as detected via } \\
\text { SARS-CoV-2 } \\
\text { nucleic acid test } \\
\text { after CP therapy, } \\
\text { third patient } \\
\text { developed } \\
\text { anaphylaxis and } \\
\text { dropped out. }\end{array}$ \\
\hline $\begin{array}{l}\text { Shen } \\
\text { et al. } \\
2020 \\
{[13]}\end{array}$ & $\begin{array}{l}\text { All patients } \\
\text { alive at the } \\
\text { time of } \\
\text { follow-up }\end{array}$ & $\begin{array}{l}32,33,35 \text { days } \\
\text { for three patients } \\
\text { (only three } \\
\text { patients } \\
\text { followed) }\end{array}$ & $\begin{array}{l}\text { Not clear, } \\
\text { probably three } \\
\text { discharged }\end{array}$ & $\begin{array}{l}\text { CRP, II-6, and } \\
\text { procalcitonin levels } \\
\text { dropped significantly } \\
\text { on day } 12 \text { post- } \\
\text { transfusion. }\end{array}$ & $\begin{array}{l}\mathrm{PAO} 2 / \mathrm{FIO} 2 \text { ranged from } 172- \\
276 \text { pre-transfusion and } \\
\text { improved to } 284-366 \text { on the } \\
\text { day } 12 \text { post-transfusion Body } \\
\text { temperature ranged from } \\
37.6-39.0^{\circ} \mathrm{C} \text { pre-transfusion } \\
\text { and became normal on the } \\
\text { third day post-transfusion. }\end{array}$ & Not reported & $\begin{array}{l}\text { CT value became } \\
\text { negative for all } \\
\text { patients on day } 12 \\
\text { post-transfusion, }\end{array}$ \\
\hline \multirow[t]{2}{*}{$\begin{array}{l}\text { Tan et } \\
\text { al. } \\
2020 \\
{[14]}\end{array}$} & Not reported & Not reported & $\begin{array}{l}\text { Probably all } \\
\text { discharged }\end{array}$ & Not reported & Not reported & Not reported & $\begin{array}{l}\text { Oropharyngeal } \\
\text { swab became } \\
\text { negative on the } \\
\text { fourth day of } \\
\text { transfusion. }\end{array}$ \\
\hline & & & & & & Resolution of & \\
\hline
\end{tabular}




\section{Cureus}

\begin{tabular}{|c|c|c|c|c|c|c|c|}
\hline $\begin{array}{l}\text { Ye et } \\
\text { al.2020 } \\
{[15]}\end{array}$ & $\begin{array}{l}\text { All patients } \\
\text { alive at the } \\
\text { time of } \\
\text { follow-up }\end{array}$ & $\begin{array}{l}4,6,6,10 \text { for four } \\
\text { patients (unclear } \\
\text { for one patient) }\end{array}$ & $\begin{array}{l}\text { Five } \\
\text { discharged }\end{array}$ & $\begin{array}{l}\text { Improvement in SARS- } \\
\text { COV-2 IgM and IgG } \\
\text { titer following CP } \\
\text { therapy. }\end{array}$ & $\begin{array}{l}5 / 6 \text { patients reported } \\
\text { improvement in shortness of } \\
\text { breath and oxygen } \\
\text { requirements. }\end{array}$ & $\begin{array}{l}\text { ground glass } \\
\text { opacifications } \\
\text { for } 5 / 6 \\
\text { patients on } \\
\text { repeat CT } \\
\text { scans } \\
\text { following CP } \\
\text { therapy. }\end{array}$ & $\begin{array}{l}\text { Throat COVID } \\
\text { swabs negative for } \\
5 / 6 \text { patients } \\
\text { following } \mathrm{CP} \\
\text { therapy }\end{array}$ \\
\hline $\begin{array}{l}\text { Zhang } \\
\text { et al. } \\
2020 \\
{[16]}\end{array}$ & $\begin{array}{l}\text { All patient } \\
\text { alive at the } \\
\text { time of } \\
\text { follow-up, } \\
\text { one patient in } \\
\text { ICU }\end{array}$ & $\begin{array}{l}7,25,27 \text { (three } \\
\text { patients } \\
\text { followed) }\end{array}$ & $\begin{array}{l}\text { Three } \\
\text { discharged }\end{array}$ & Not reported & $\begin{array}{l}\text { Improvement in oxygen } \\
\text { saturation. }\end{array}$ & $\begin{array}{l}\text { Significant } \\
\text { improvement } \\
\text { in pulmonary } \\
\text { infiltrates } \\
\text { noted on } \\
\text { repeat } \\
\text { imaging } \\
\text { (chest } \\
\text { radiographs } \\
\text { and CT } \\
\text { scans). }\end{array}$ & $\begin{array}{l}\text { RT-PCR and } \\
\text { oropharyngeal } \\
\text { swabs noted to be } \\
\text { negative. }\end{array}$ \\
\hline $\begin{array}{l}\text { Zhang } \\
\text { et al. } \\
2020 \\
{[17]}\end{array}$ & Not reported & Not reported & $\begin{array}{l}\text { Probably all } \\
\text { discharged }\end{array}$ & Not reported & $\begin{array}{l}\text { Improvement in ventilation } \\
\text { status with patient not } \\
\text { requiring mechanical } \\
\text { ventilation on day } 11 \text { of } \mathrm{CP} \\
\text { therapy. }\end{array}$ & Not reported & Not reported \\
\hline $\begin{array}{l}\text { Zeng } \\
\text { et al. } \\
2020 \\
{[18]}\end{array}$ & $\begin{array}{l}\text { Five patients } \\
\text { died. No } \\
\text { changes in } \\
\text { mortality } \\
\text { noted with } \\
\text { the use of CP. }\end{array}$ & Not reported & $\begin{array}{l}\text { One } \\
\text { discharged }\end{array}$ & Not reported & Not reported & Not reported & $\begin{array}{l}\text { RT-PCR and } \\
\text { oropharyngeal } \\
\text { swabs noted to be } \\
\text { negative for all } \\
\text { patients. }\end{array}$ \\
\hline $\begin{array}{l}\text { Li et al. } \\
2020 \\
{[19]}\end{array}$ & $\begin{array}{l}\text { No } \\
\text { statistically } \\
\text { significant } \\
\text { difference in } \\
28 \text {-day } \\
\text { mortality in } \\
\text { treatment vs. } \\
\text { control group } \\
(15.7 \% \text { vs } \\
24.0 \% ; \\
\mathrm{p}=0.30),\end{array}$ & $\begin{array}{l}\text { No statistically } \\
\text { significant } \\
\text { difference time to } \\
\text { discharge on day } \\
28 \text { of follow up } \\
(51.0 \% \text { in } \\
\text { treatment vs } \\
36.0 \% \text { in the } \\
\text { control group } \\
\mathrm{p}=0.12)\end{array}$ & $\begin{array}{l}21 / 23(91.3) \\
\text { and } 15 / 22 \\
\text { (68.2) patients } \\
\text { discharged in } \\
\text { the treatment } \\
\text { and control } \\
\text { group } \\
\text { respectively on } \\
\text { day } 28 \text { of } \\
\text { follow up. }\end{array}$ & Not reported & $\begin{array}{l}\text { No statistically significant } \\
\text { clinical improvement } \\
\text { achieved on day } 28 \text { of follow- } \\
\text { up (51.9\% [27/52] patients } \\
\text { improved the convalescent } \\
\text { plasma group vs } 43.1 \% \\
(22 / 51) \text { in the control group; } \\
\mathrm{p}=0.26) \text {. }\end{array}$ & Not reported & $\begin{array}{l}\text { SARS-CoV-2 viral } \\
\text { PCR reported } \\
\text { negative earlier } \\
\text { compared to the } \\
\text { control group } \\
(87.2 \% \text { treatment } \\
\text { group vs } 37.5 \% \\
\text { control group; } \\
\mathrm{p}<0.001 \text {. }\end{array}$ \\
\hline
\end{tabular}

\section{TABLE 2: Table summarizing treatment outcomes following convalescent plasma therapy}

ICU - intensive care unit, CRP - C-reactive protein; IL-6 - interleukin 6; CP - convalescent plasma; RT-PCR - reverse transcription polymerase chain reaction; $\mathrm{PaO} 2 / \mathrm{FiO} 2$ - partial pressure of oxygen in arterial blood/fraction of inspired oxygen.

\section{Discussion}

The randomized evaluation of COVID-19 therapy (RECOVERY) trial is the only large scale trial suggesting dexamethasone as an effective treatment for reducing COVID-19 mortality in critically ill patients [20]. Despite the acceleration of the COVID-19 spread, we are still struggling to find a concrete treatment. Therefore, our systematic review is valuable as it explores the current literature and aims at assessing the efficacy of convalescent plasma therapy for treating COVID-19.

Plasma therapy has long been used for the treatment of infectious diseases such as Ebola, MERS, and SARS [5-8]. Schoofs et al. suggested that antibodies in convalescent plasma suppresses viremia and tested 3BNC117 antibody for its ability to suppress HIV-1 viremia. 3BNC117 is a potent antibody that binds to the CD4 binding sites on the viral envelope. Even after a single passive administration in animal models, Schoof et al. noted the antibody to suppress HIV-1 viremia [20]. In-vivo studies also suggest that antibodies not only reduce the viral load and reduce the rate of infection of new cells but increase the clearance rate of existing infected cells as well [21]. 
Our systematic review noted that there was no standardization in terms of the time of administration of plasma therapy. Existing research suggests that SARS viral viremia peaks during the first week of infection and patients usually start to develop primary immune response by the end of the second week of their infection. Therefore, the administration of plasma early during the early stage of the disease might lead to more favorable clinical outcomes [22].

Most of the studies included in our systematic review showed that convalescent plasma therapy leads to an improvement in clinical outcomes. However, the only RCT by Li et al. showed that the patients receiving CP did not differ from control groups on the six-point clinical severity scale on the 28th day of follow-up [19]. Furthermore, almost all patients were discharged in the rest of the studies by the only RCT by Li et al. noted that the mortality did not change significantly between CP and control groups [19].

Limitations

The results of the available research should be interpreted with great caution. The available data suggesting positive effects of CP on patients' clinical symptoms and mortality mainly come from case reports and case series that lack randomization, have a limited data set, and have a high risk of bias. The only available RCT suggests otherwise and does not report any changes in mortality and improvement in clinical symptoms with the use of CP. Furthermore, it must also be noted that the use of convalescent plasma for COVID-19 has significant clinical and practical limitations. As noted in previous studies, patients recovering from SARS infection require at least 12 weeks for their IgG neutralizing antibody titer (NAT) to reach $\geqslant 1: 160$ and only the $\mathrm{CP}$ that had a NAT of $\geqslant 1: 160$ reduced mortality in SARS cases [23]. Moreover, limitations such as getting informed consent from the donors and recipients, state of health of donor and recipient, the amount of plasma acquired from one donor, and the mismatch of the number of donors versus the patients who need this therapy may significantly limit the clinical utility of CP for treating COVID-19 cases [24]. Also, adverse reactions such as transfusion-related anaphylactic reactions, the transmission of infections, and other adverse events such as fever, chills, and lung injury are valid clinical concerns that should not be overlooked [25].

\section{Conclusions}

COVID-19 is a global pandemic with no proven treatment. The changing situation is posing a serious therapeutic dilemma for the clinicians and there is an urgent need for therapies that could help reduce patient mortality. Amidst the therapeutic uncertainties, convalescent plasma therapy might have some therapeutic potential. Our systematic review shows that plasma therapy might produce a notable improvement in patient symptoms and clinical and biochemical parameters associated with COVID-19 infection. Although there is some preliminary evidence that plasma therapy might improve patient mortality but this fact needs to be validated through organized RCTs. Despite the potential benefits, plasma therapy has significant limitations such as lack of availability, a dearth of standardization of this treatment method, and paucity of compelling clinical evidence advocating its use. Despite these limitations, the early use of convalescent plasma therapy may be considered as an adjuvant for critically-ill COVID-19 patients.

\section{Additional Information \\ Disclosures}

Conflicts of interest: In compliance with the ICMJE uniform disclosure form, all authors declare the following: Payment/services info: All authors have declared that no financial support was received from any organization for the submitted work. Financial relationships: All authors have declared that they have no financial relationships at present or within the previous three years with any organizations that might have an interest in the submitted work. Other relationships: All authors have declared that there are no other relationships or activities that could appear to have influenced the submitted work.

\section{References}

1. World Health Organization: coronavirus disease 2019 (COVID-19) - Situation Report 175 . (2020). Accessed: July 13, 2020: https://www.who.int/docs/default-source/coronaviruse/situation-reports/20200713-covid-19sitrep-175.pdf.

2. Baud D, Qi X, Nielsen-Saines K, Musso D, Pomar L, Favre G: Real estimates of mortality following COVID19 infection. Lancet Infect Dis. 2020, 12:773. 10.1016/S1473-3099(20)30195-X

3. Nicola M, O'Neill N, Sohrabi C, Khan M, Agha M, Agha R: Evidence based management guideline for the COVID-19 pandemic - review article. Int J Surg. 2020, 77:206-216. 10.1016/j.ijsu.2020.04.001

4. Marano G, Vaglio S, Pupella S, et al.: Convalescent plasma: new evidence for an old therapeutic tool? . Blood Transfus. 2016, 14:152-157. 10.2450/2015.0131-15

5. Griensven J, Edwards T, de Lamballerie X, et al.: Evaluation of convalescent plasma for Ebola virus disease in Guinea. N Engl J Med. 2016, 374:33-42. 10.1056/NEJMoa1511812

6. Hung I, To K, Lee C, et al.: Convalescent plasma treatment reduced mortality in patients with severe pandemic influenza A (H1N1) 2009 virus infection. Clin Infect Dis. 2011, 15:447-456. 10.1093/cid/ciq106

7. The WHO MERS-CoV Research Group: State of knowledge and data gaps of Middle East Respiratory Syndrome Coronavirus (MERS-CoV) in humans. PLoS Curr. 2013, 12:5.

10.1371/currents.outbreaks.0bf719e352e7478f8ad85fa30127ddb8 
8. Wong V, Dai D, Wu A, Sung J: Treatment of severe acute respiratory syndrome with convalescent plasma . Hong Kong Med J. 2003, 9:199-201.

9. Valk S, Piechotta V, Chai K, et al.: Convalescent plasma or hyperimmune immunoglobulin for people with COVID- 19: a rapid review. Cochrane Database Syst Rev. 2020, 5:013600. 10.1002/14651858.CD013600

10. Ahn J, Sohn Y, Lee S, et al.: Use ofconvalescent plasma therapy in two COVID-19 patients withacute respiratory distress syndrome in Korea. J Korean Med Sci. 2020, 35:e149. 10.3346/jkms.2020.35.e149

11. Duan K, Liu B, Li C, et al.: Effectiveness of convalescent plasma therapy in severe COVID-19 patients . Proc Natl Acad Sci USA . 2020, 28:9490-9496. 10.1073/pnas.2004168117

12. Pei S, Yuan X, Zhimin Z, et al.: Convalescent plasma to treat COVID- 19: Chinese strategy andexperiences (PREPRINT). medRxiv. 2020, 10.1101/2020.04.07.20056440

13. Shen C, Wang Z, Zhao F, et al.: Treatment of 5 critically ill patients with COVID-19 with convalescent plasma. JAMA. 2020, 323:1582-1589. 10.1001/jama.2020.4783

14. Tan L, Kang X, Zhang B, et al.: A special case of COVID-19 with long duration of viral shedding for 49 days (PREPRINT). medRxiv. 2020, 10.1101/2020.03.22.20040071

15. Ye M, Fu D, Ren Y, et al.: Treatment with convalescent plasma for COVID-19 patients in Wuhan, China (IN PRESS). J Med Virol. 2020, 10.1002/jmv.25882

16. Zhang B, Liu S, Tan T, et al.: Treatment with convalescent plasma for critically ill patients with SARS-CoV2 infection. Chest. 2020, 31:E9-E13. 10.1016/j.chest.2020.03.039

17. Zhang L, Pang R, Xue X, et al.: Anti-SARS-CoV-2virus antibody levels in convalescent plasma of six donors whohave recovered from COVID-19. Aging. 2020, 12:6536-6542. 10.18632/aging.103102

18. Zeng Q, Yu Z, Gou J, et al.: Effect of convalescent plasma therapy on viral shedding and survival in patients with coronavirus disease. J Infect Dis. 2020, 222:38-43. 10.1093/infdis/jiaa228

19. Li L, Zhang W, Hu Y, et al.: Effect of convalescent plasma therapy on time to clinical improvement in patients with severe and life-threatening COVID-19. JAMA. 2020, 10.1001/jama.2020.10044

20. Schoofs T, Klein F, Braunschweig M: HIV-1 therapy with monoclonal antibody 3BNC117 elicits host immune responses against HIV-1. Science. 2016, 352:997-1001. 10.1126/science.aaf0972

21. Lu C, Murakowski D, Bournazos S: Enhanced clearance of HIV-1-infected cells by broadly neutralizing antibodies against HIV-1 in vivo. Science. 2016, 352:1001-1004. 10.1126/science.aaf1279

22. Cheng Y, Wong R, Soo Y, et al.: Use of convalescent plasma therapy in SARS patients in Hong Kong . Eur J Clin Microbiol Infect Dis. 2005, 24:44-46. 10.1007/s10096-004-1271-9

23. Hung I, To K, Lee C: Convalescent plasma treatment reduced mortality in patients with severe pandemic influenza A (H1N1) 2009 virus infection. Clin Infect Dis. 2011, 52:447-456. 10.1093/cid/ciq106

24. Zhao Q, He Y: Challenges of Convalescent Plasma Therapy on COVID-19 . J Clin Virol. 2020, 127:104358. 10.1016/j.jcv.2020.104358

25. MacLennan S, Barbara J: Risks and side effects of therapy with plasma and plasma fractions . Best Pract Res Clin Haematol. 2006, 19:169-189. 10.1016/j.beha.2005.01.033 